\title{
PROJETO DE UM TRANSDUTOR ULTRASSÔNICO FLANGEADO PARA APLICAÇÃO EM LIMPEZA DE DUTOS
}

\section{Evilazio Coelho Soares 1; Luiz Carlos Simões Soares Júnior 2²; luri Muniz Pepe $3^{2}$}

${ }^{1}$ Laboratório de Propriedades Óticas, Universidade Federal da Bahia, Rua Barão de Jeremoado, S/N, Campus de Ondina, CEP.: 40170-115; Salvador/Bahia/Brasil; evilaziocoelho03@gmail.com

${ }^{2}$ Laboratório de Propriedades Óticas, Universidade Federal da Bahia, Rua Barão de Jeremoado, S/N, Campus de Ondina, CEP.: 40170-115; Salvador/Bahia/Brasil

Resumo: O objetivo deste trabalho é desenvolver um transdutor que execute a tarefa de limpeza de dutos de transporte de óleo. Pesquisa bibliográfica sobre efeito piezoelétrico e transdutores ultrassônicos foi feita. Dedução matemática sobre modelagem de elementos eletromecânicos e mecânicos, componentes do transdutor, foi realizada. Ferramenta computacional foi utilizada para resolver a modelagem do transdutor. Experimento no transdutor com equipamento de análise de curva de impedância foi efetuado. A frequência de ressonância do modelo teórico foi $39,24 \mathrm{kHz}$. No caso experimental, a ressonância do transdutor desenvolvido foi de $37,63 \mathrm{kHz}$. Por fim, a validade do modelo foi provada.

Palavras-Chave: transdutor; ultrassom; instrumentação; modelagem.

\section{DESIGN OF A FLANGED ULTRASONIC TRANSDUCER FOR PIPE CLEANING APPLICATION}

\begin{abstract}
The objective of this work is to develop a transducer that performs the task of cleaning oil transport ducts. Bibliographic research on piezoelectric effect and ultrasonic transducers was made. Mathematical deduction on modeling of electromechanical and mechanical elements, transducer components, was performed. Computational tool was used to solve the transducer modeling. Transducer experiment with impedance curve analysis equipment was performed. The resonant frequency of the theoretical model was $39.24 \mathrm{kHz}$. In the experimental case, the developed transducer resonance was $37.63 \mathrm{kHz}$. Finally, the validity of the model has been proven.
\end{abstract}

Keywords: transducer; ultrasound; instrumentation; modeling. 


\section{INTRODUÇÃO}

As cerâmicas piezoelétricas surgiram na década de cinquenta visando aplicações em sonares [1]. O efeito piezoelétrico foi descoberto em 1880 em cristais de quartzo pelos irmãos Pierre e Jacques Curie. Desde então tem motivado inúmeras investigações para 0 desenvolvimento de sistemas transdutores eletromecânicos [1].

O efeito piezoelétrico consiste basicamente na conversão de energia mecânica em elétrica (do grego "piezo" -> "pressão"). Posteriormente, em 1881, por análises termodinâmicas, Lippman previu a existência do "efeito piezoelétrico inverso", que consiste no aparecimento de uma deformação do material quando submetido a um campo elétrico [1].

A primeira aplicação tecnológica de um elemento piezoelétrico pode ser atribuída a Langevin que, em 1921, desenvolveu um sonar utilizando o quartzo como elemento piezoelétrico [1].

Ultrassom são ondas sonoras com frequências localizadas acima do limite audível para o ser humano (acima de $16 \mathrm{KHz}$ ). Para os propósitos de obtenção de imagens (ultrassonografia), por exemplo, freqüências entre 1 e $10 \mathrm{MHz}$ são usadas. O ultrassom, geralmente, se propaga por líquidos, tecidos e sólidos [2].

Laboratório de Propriedades Óticas (LaPO) do Instituto de Física da UFBA possui diversos projetos que tratam do uso de transdutores piezoelétricos de ultrassom em tubulações. O objetivo geral deste trabalho é desenvolver um transdutor com flange que possa ser conectado a dutos de transporte de óleo para executar a tarefa de limpeza.

\section{REVISÃO DA LITERATURA}

\subsection{Efeito Piezelétrico e Transdutores Ultrassônicos}

Transdutores são dispositivos que transformam uma forma de energia em outra. Portanto, transdutores ultrassônicos de potência são dispositivos que convertem energia elétrica em vibrações mecânicas de alta frequência e intensidade [3]. Uma pastilha piezoelétrica é um elemento que gera um campo elétrico a partir da aplicação de uma tensão mecânica. Por outro lado, quando um campo elétrico é aplicado nessa pastilha, ocorre a geração de deformação mecânica. Os materiais piezoelétricos geram carga elétrica proporcional à aplicação da tensão mecânica e também, de maneira contrária, exibem uma deformação mecânica proporcional ao campo elétrico aplicado.

O Transdutor Langevin ou Transdutor Sanduíche ultrassônico é uma estrutura ressonante de meia onda que utiliza uma alta tensão de alta frequência para gerar vibrações mecânicas, com a ajuda de efeito piezoelétrico. Quando à estrutura, o transdutor ultrassônico piezoelétrico Langevin possui duas massas de carga que estão ligadas em duas superfícies planas de uma piezocerâmica [4,5].

Dependendo da aplicação, o transdutor é conectado a um amplificador mecânico, expansor, que aumenta a amplitude de deslocamento em sua face final. Em aplicações de ultrassom de potência, o transdutor tipo Langevin e o amplificador 
mecânico são projetados para operar na mesma frequência de ressonância, formando um conjunto sintonizado [6].

No transdutor estudado neste trabalho, as cerâmicas PZT (Titanato Zirconato de Chumbo) são presas entre a massa traseira e o expansor por conexão parafusada. São utilizadas duas cerâmicas piezoelétricas polarizadas longitudinalmente em direções opostas. Quando a voltagem alternada é aplicada nos eletrodos, a vibração longitudinal é gerada. Uma amplitude de vibração maior pode ser observada na frequência de ressonância $[5,7,8]$.

\subsection{Modelagem dos elementos eletromecânicos (cerâmica piezoelétrica)} Figura 1.

Baseado em [3], assume-se uma pastilha piezoelétrica anelar como a da

Figura 1. Cerâmica piezoelétrica, geometria e eixos de referência $(A)$; Representação de um elemento infinitesimal da cerâmica piezoelétrica (B). Fonte: Adaptado de [3].

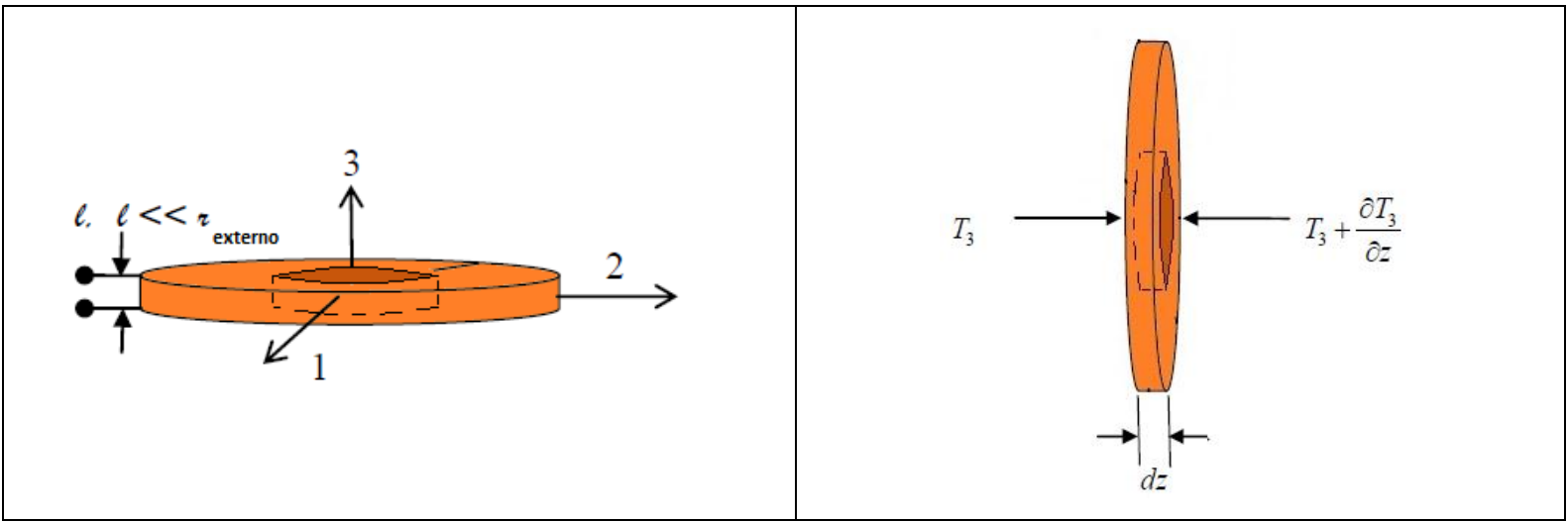

As equações constitutivas para a cerâmica piezelétrica são:

$$
\begin{aligned}
& T_{3}=c_{33}^{E} S_{3}-e_{33} E_{3} \\
& D_{3}=e_{33} S_{3}+\varepsilon_{33}^{S} E_{3}
\end{aligned}
$$

onde $T_{3}$ é a tensão mecânica na direção $3 ; S_{3}$ é a deformação mecânica na direção 3; $c_{33}^{E}$ é a rigidez elástica no plano 33 medida com campo elétrico constante; $e_{33}$ é o coeficiente piezoelétrico no plano $33 ; \varepsilon_{33}^{S}$ é a permissividade elétrica no plano 33 obtida a uma deformação constante; e $D_{3}$ é a densidade de carga elétrica na direção 3.

Considerando um elemento infinitesimal de cerâmica da Figura 1B, de espessura $d z$, submetida a forças que geram tensões mecânicas na superfície da cerâmica, $\rho$ é a densidade da cerâmica piezoelétrica utilizada no transdutor, $t$ corresponde ao instante de tempo, tem-se da aplicação da segunda lei de Newton no elemento:

$$
\rho \frac{\partial^{2} u_{3}}{\partial t^{2}}=\frac{\partial T_{3}}{\partial z}
$$


em que $u_{3}$ é a vibração longitudinal $\left(S_{3}=\frac{\partial T u_{3}}{\partial z}\right)$, propagando-se na direção do eixo 3; e $T_{3}$ é a tensão mecânica na direção 3.

Sendo a rigidez elástica no plano $33, c_{33}^{D}$, medida com polarização constante dada pela equação (04).

$$
c_{33}^{D}=c_{33}^{E}+\frac{e_{33}^{2}}{\varepsilon_{33}^{S}}
$$

Depois de algumas substituições matemáticas, obtém-se a seguinte expressão:

$$
\frac{\partial^{2} u_{3}}{\partial t^{2}}-\frac{c_{33}^{D}}{\rho} \frac{\partial^{2} u_{3}}{\partial z^{2}}=0
$$

Resolvendo a equação (05), tem-se a seguinte equação diferencial em $z$, onde $k$ é o número de onda da cerâmica.

$$
\frac{\partial^{2} u_{3}(z)}{\partial z^{2}}+k^{2} u_{3}(z)=0
$$

Após determinar as condições de contorno nas extremidades da cerâmica:

$$
\begin{gathered}
T_{3}(0)=-\frac{F_{1}}{A_{s}}, \\
T_{3}(l)=-\frac{F_{2}}{A_{s}} \\
\frac{\partial u_{3}(0)}{\partial t}=v_{1}, \\
\frac{\partial u_{3}(l)}{\partial t}=-v_{2}
\end{gathered}
$$

Obtém-se a solução da equação diferencial na variável $z$, em que $v_{1}$ e $v_{2}$ são as velocidades nas faces, $\omega$ é a velocidade angular e $l$ corresponde à espessura da cerâmica.

$$
u_{3}(z)=\frac{1}{j \omega}\left[-\frac{v_{2}+v_{1} \cos (k l)}{\sin (k l)} \sin (k z)+v_{1} \cos (k z)\right]
$$

Assim, obtém-se o vetor densidade de corrente na direção $z$ :

$$
D_{3}(z)=-\frac{e_{33} k}{j \omega}\left[v_{1} \sin (k z)+\frac{v_{2}+v_{1} \cos (k l)}{\sin (k l)} \cos (k z)\right]+\varepsilon_{33}^{S} E_{3}(z)
$$

Aplicando a lei de Gauss para determinar a intensidade de corrente que circula na cerâmica piezoelétrica e substituindo a expressão obtida, tem-se o campo elétrico:

$$
E_{3}(z)=\frac{I}{j \omega A_{s} \varepsilon_{33}^{S}}+\frac{e_{33}}{j \omega \varepsilon_{33}^{S}}\left[v_{1} \sin (k z)+\frac{v_{2}+v_{1} \cos (k l)}{\sin (k l)} \cos (k z)\right]
$$

A tensão elétrica é obtida pela integração do campo elétrico, que resulta em:

$$
V=\frac{I l}{j \omega A_{s} \varepsilon_{33}^{S}}+\left[\frac{e_{33}}{j \omega \varepsilon_{33}^{S} k}(1-\cos (k l)]+\frac{e_{33}}{j \omega \varepsilon_{33}^{S} k}\left[v_{2}+v_{1} \cos (k l)\right]\right.
$$

Utilizando as condições de contorno para determinar as forças nas faces da cerâmica e fazendo todas as substituições, tem-se que: 


$$
\begin{gathered}
F_{1}=\frac{Z_{A}}{j \tan k l} v_{1}+\frac{Z_{A}}{j \sin k l} v_{2}+\frac{e_{33}}{j \omega e_{33}} I \\
F_{2}=\frac{Z_{A}}{j \sin k l} v_{1}+\frac{Z_{A}}{j \tan k l} v_{2}+\frac{e_{33}}{j \omega e_{33}} I \\
V=\frac{e_{33}}{j \omega \varepsilon_{33}^{S}} v_{1}+\frac{e_{33}}{j \omega \varepsilon_{33}^{S}} v_{2}+\frac{I}{j \omega e C_{0}}
\end{gathered}
$$

onde $Z_{A}=\rho c_{c} A_{S}$ (impedância elétrica) e $C_{0}=\varepsilon_{33}^{S} A_{S} / l$ (capacitância elétrica); $c_{c}$ é a velocidade de propagação extensional da onda na cerâmica e $A_{s}$ é a área superficial.

Fazendo substituições e agrupamentos, chega-se à forma matricial

$$
\left(\begin{array}{l}
v_{2} \\
F_{2} \\
V
\end{array}\right)=\left(\begin{array}{lll}
A_{11} & A_{12} & A_{13} \\
A_{21} & A_{22} & A_{23} \\
A_{31} & A_{32} & A_{33}
\end{array}\right)\left(\begin{array}{c}
v_{1} \\
F_{1} \\
I
\end{array}\right)
$$

em que cada elemento da matriz de ordem 3 é obtido pela manipulação dos termos das equações (15), (16) e (17).

\subsection{Modelagem dos Elementos Mecânicos}

Ainda segundo [3], as massas metálicas dos transdutores de potência também são modeladas. Para tanto é utilizada a equação de onda no meio elástico:

$$
\frac{\partial^{2} u_{m}}{\partial t^{2}}=c_{m}^{2} \frac{\partial^{2} u_{m}}{\partial z^{2}}
$$

em que $c_{m}$ é a velocidade de propagação extensional da onda no elemento mecânico; $u_{m}$ é o deslocamento na direção axial $z$.

Propondo resolução de equação diferencial por separação de variáveis e obtém-se a seguinte equação diferencial na variável $z$ :

$$
\frac{\partial^{2} u_{m}}{\partial z^{2}}+k_{m}^{2} u_{m}=0
$$

onde $k_{m}$ é número de onda do elemento mecânico. mecânico:

Após determinar as condições de contorno nas extremidades do elemento

$$
\begin{gathered}
T_{3}(z=0)=-\frac{F_{1}}{A_{m}}, \\
T_{3}(z=l)=-\frac{F_{2}}{A_{m}}, \\
\frac{\partial u_{m}}{\partial t}(z=0)=v_{1}, \\
\frac{\partial u_{m}}{\partial t}(z=l)=v_{2}
\end{gathered}
$$

Obtém-se a solução da equação diferencial na variável $z$ :

$$
u_{m}(z, t)=\left[-\frac{F_{1}}{c_{m}^{2} \rho_{m} A_{m} k_{m}} \sin \left(k_{m} z\right)+\frac{v_{1}}{j \omega} \cos \left(k_{m} z\right)\right] e^{j \omega t}
$$


E obtém-se a seguinte notação matricial:

$$
\left(\begin{array}{c}
v_{2} \\
F_{2}
\end{array}\right)=\left(\begin{array}{cc}
\cos \left(k_{m} l\right) & -\frac{j}{Z_{m}} \sin \left(k_{m} l\right) \\
\frac{Z_{m}}{j} \sin \left(k_{m} l\right) & \cos \left(k_{m} l\right)
\end{array}\right)\left(\begin{array}{l}
v_{1} \\
F_{1}
\end{array}\right)
$$

\section{MATERIAIS E MÉTODOS}

O transdutor proposto possui uma massa traseira cilíndrica em aço inox. Ele é composto de duas pastilhas piezelétricas do tipo PZT-4 conectadas em série entre si. O flange para acoplamento do transdutor ao duto possui $95 \mathrm{~mm}$ de diâmetro e 0 horn é do tipo stepped com $35 \mathrm{~mm}$ de diâmetro. A Figura 2 exibe o modelo 3D e a foto do transdutor com o flange.

Figura 2. Modelo 3D (A) e foto do transdutor com flange (B). Fonte: Autoria própria.

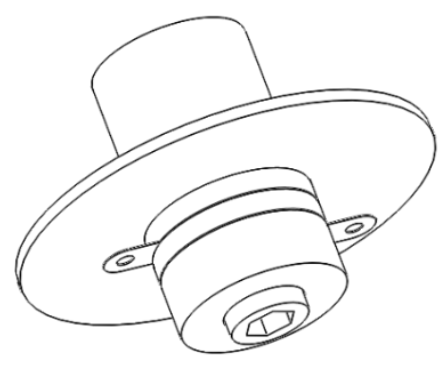

A

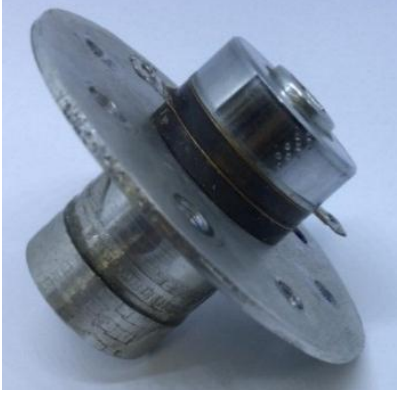

$\mathrm{B}$

As especificações dos elementos mecânicos e eletromecânicos são apresentadas na Tabela 1.

Tabela 1. Especificações técnicas dos elementos mecânicos e eletromecânicos.

\begin{tabular}{|l|c|c|c|c|}
\hline \multirow{2}{*}{ Especificação } & \multicolumn{4}{c|}{ Elementos } \\
\cline { 2 - 5 } & Massa traseira & Flange & Horn & Cerâmica \\
\hline Material & Aço Inox & Alumínio & Alumínio & PZT-4 \\
\hline Raio externo $(\mathrm{mm})$ & 19,0 & 47,5 & 17,5 & 19,0 \\
\hline Raio interno $(\mathrm{mm})$ & 7,6 & 7,6 & 0,0 & 7,6 \\
\hline Espessura $(\mathrm{mm})$ & 13,0 & 2,7 & 36,0 & 5,0 \\
\hline Densidade $\left(\mathrm{kg} / \mathrm{m}^{3}\right)$ & 7860 & 2700 & 2700 & 7700 \\
\hline Velocidade do som $\left(\mathrm{m} / \mathrm{s}^{2}\right)$ & 5950 & 6420 & 6420 & 3820 \\
\hline Permissividade & - & - & - & $5,2 \mathrm{E}-9$ \\
\hline Fator de qualidade mecânica & - & - & - & 600 \\
\hline
\end{tabular}

\section{RESULTADOS E DISCUSSÃO}

Foram desenvolvidas as matrizes para cada um dos elementos mecânicos e eletromecânicos baseadas nas equações (15), (16), (17) e na notação matricial (26). Foi utilizada a ferramenta computacional Matlab para resolver o produto das matrizes, determinar a frequência de ressonância e o deslocamento máximo do transdutor. Os resultados são apresentados na Figura 3 para a faixa de frequências entre 20 e $50 \mathrm{kHz}$.

Figura 3. Curva de impedância $(A)$ e deslocamento máximo $(B)$ do modelo de transdutor proposto. Fonte: Autoria própria. 


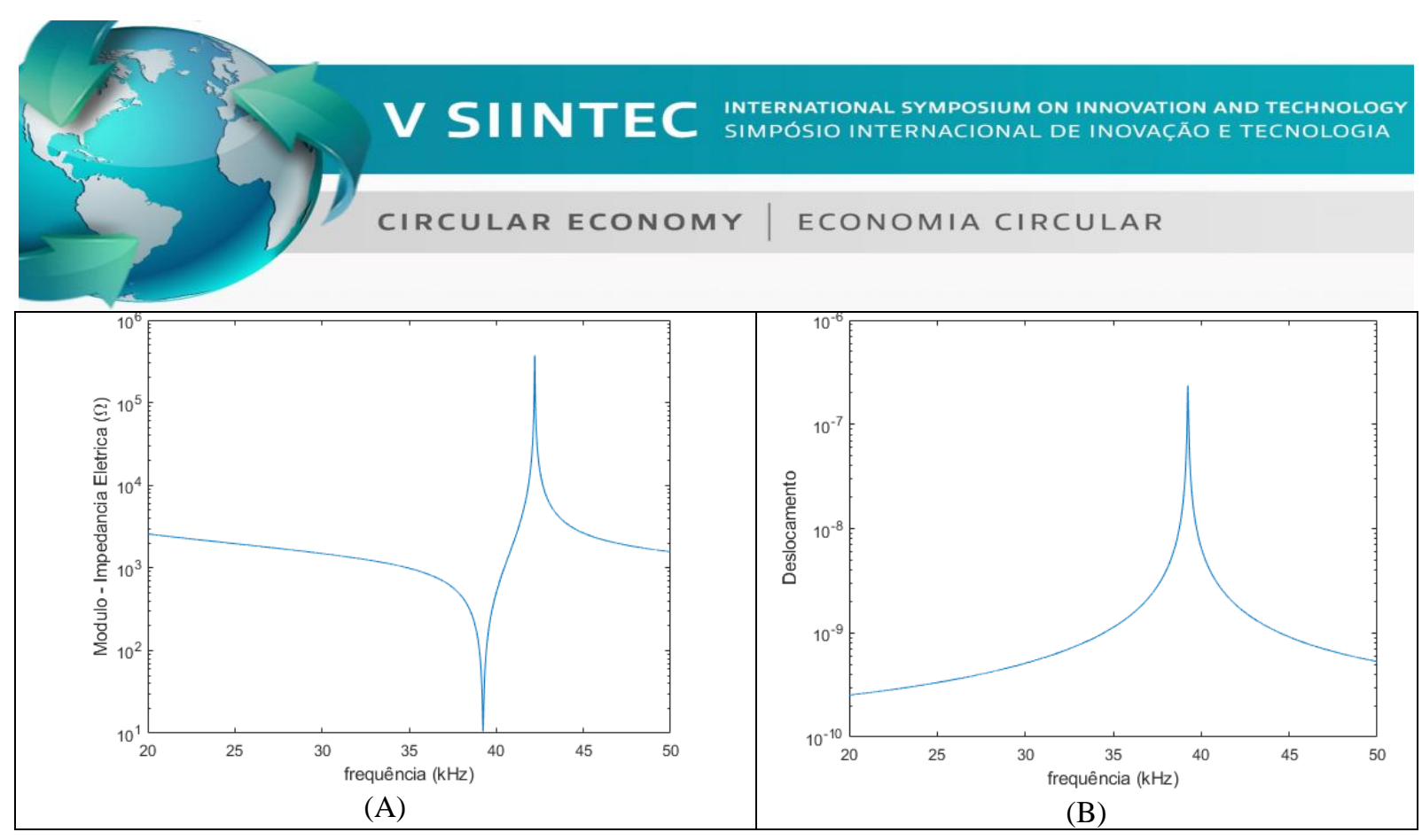

Assim, pela Figura 3, o transdutor proposto apresentou frequência de ressonância em $39,24 \mathrm{kHz}$, onde apresentou impedância de 10,55 $\Omega$. O seu deslocamento máximo foi de $0,235 \mu \mathrm{m}$ para a frequência de $39,24 \mathrm{kHz}$.

Para avaliar o modelo proposto foi desenvolvido o transdutor, ele teve suas características avaliadas em equipamento de análise de curva de impedância (ultrasonic transducer and horn analyzer), o TRZ fabricado pela ATCP. A curva de impedância do transdutor desenvolvido é apresentada na Figura 4.

Figura 4. Curva de impedância do transdutor flangeado desenvolvido. Fonte: Autoria própria.

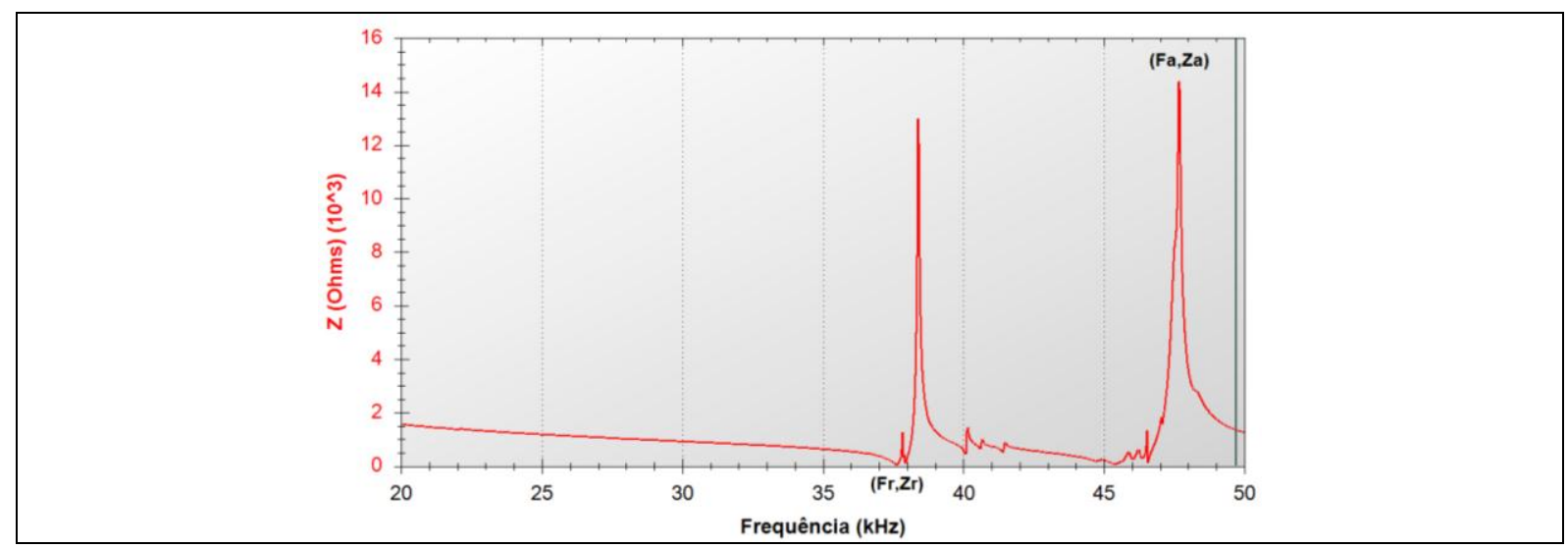

Com base no gráfico de impedância obtido pelo TRZ, Figura 4, a ressonância do transdutor desenvolvido foi de $37,63 \mathrm{kHz}$ onde foi obtida uma impedância de 47,5 $\Omega$. A discrepância entre os valores teórico e experimental de ressonância foi de 4,3 $\%$. Apesar de uma grande diferença entre os valores experimentais e teóricos obtidos para a impedância, o resultado obtido experimentalmente ainda pode ser considerado bom, uma vez que o modelo teórico não leva em consideração as perdas de energia associadas ao acoplamento entre as peças.

\section{CONCLUSÃO}


Neste trabalho foram apresentadas informações sobre 0 transdutor piezoelétrico de ultrassom principalmente sobre o Transdutor Langevin. Foi descrito um histórico de como surgiu tal dispositivo bem como conceitos e aplicações.

O tipo de modelagem matemática pesquisado e escolhido para o transdutor é válido, visto que os resultados teórico e experimental para a frequência de ressonância apresentaram uma discrepância de 4,3 \%.

\section{REFERÊNCIAS}

${ }^{1}$ ADAMOWSKI, J. C. Sensores: Teoria e Aplicações. Laboratório de Sensores e Atuadores. Departamento de Engenharia e de Sistemas Mecânicos. Escola Politécnica da USP, 2000.

2 BISCEGLI, C. I. Conceitos da física do ultrassom. São Carlos, SP: Embrapa Instrumentação Agropecuária, USP, 2004.

3 TAYRA, V. T. Desenvolvimento de um transdutor ultrassônico de potência aplicado a perfuração de rochas e usinagem de metais. São Paulo, 2014.

4 JOVANOVIĆ, Igor; JOVANOVIĆ, Uglješa; MANČIĆ, Dragan. A Matlab/Simulink 3D Model of Unsymmetrical Ultrasonic Sandwich Transducers. Serbian Journal of Electrical Engineering, v. 15, n. 1, 41-52, 2018.

5 IULA, A. et al. A 3-D Model of the Classical Langevin Transducer. IEEE Ultrasonics Symposium, 987-990, 1997.

6 ALVAREZ, Nicolás P. et al. Nonlinear Dynamic Modeling of Langevin-Type Piezoelectric Transducers. Actuators, 4, 255-266, 2015.

7 GHENNA, Sofiane; GIRAUD, Frédéric; GIRAUD-AUDINE, Christophe; AMBERG, Michel; LEMAIRE-SEMAIL, Betty. Modelling, identification and control of a Langevin transducer. Disponível em:<https://www.researchgate.net/publication/ 2795328522015>. Acesso em: 12 fev. 2019.

8 MARUYAMA, Yutaka; TAKASAKI, Masaya; MIZUNO, Takeshi. Resonance Frequency Tracing System for Langevin Type Ultrasonic Transducers. Graduate School of Science \& Engineering. Saitama University. Japan, 2010. 